\title{
Expression patterns of Fgf8 and Shh in the developing external genitalia of Suncus murinus
}

\author{
Mami Miyado ${ }^{1}$, Kenji Miyado², Akihiro Nakamura², Maki Fukami ${ }^{1}$, Gen Yamada ${ }^{3}$ and \\ Sen-ichi Oda ${ }^{4}$ \\ ${ }^{1}$ Department of Molecular Endocrinology, ${ }^{2}$ Department of Reproductive Biology, National Research Institute for \\ Child Health and Development, Setagaya, Tokyo, Japan, ${ }^{3}$ Department of Developmental Genetics, Institute of \\ Advanced Medicine, Wakayama Medical University, Wakayama, Japan, and ${ }^{4}$ Laboratory of Animal Management \\ and Resources, Department of Zoology, Okayama University of Science, Okayama, Japan
}

Correspondence should be addressed to M Miyado; Email: miyado-m@ncchd.go.jp

\begin{abstract}
Reciprocal epithelial-mesenchymal interactions and several signalling pathways regulate the development of the genital tubercle (GT), an embryonic primordium of external genitalia. The morphology of the adult male external genitalia of the Asian house musk shrew Suncus murinus (hereafter, laboratory name: suncus) belonging to the order Eulipotyphla (the former order Insectivora or Soricomorpha) differs from those of mice and humans. However, the developmental process of the suncus GT and its regulatory genes are unknown. In the present study, we explored the morphological changes and gene expression patterns during the development of the suncus GT. Morphological observations suggested the presence of common (during the initial outgrowth) and species-specific (during the sexual differentiation of GT) developmental processes of the suncus GT. In gene expression analysis, fibroblast growth factor 8 (Fgf8) and sonic hedgehog ( $\mathrm{Sh}$ ), an indicator and regulator of GT development in mice respectively, were found to be expressed in the cloacal epithelium and the developing urethral epithelium of the suncus GT. This pattern of expression specifically in GT epithelium is similar to that observed in the developing mouse GT. Our results indicate that the mechanism of GT formation regulated by the FGF and SHH signalling pathways is widely conserved in mammals.
\end{abstract}

Reproduction (2017) 153 187-195

\section{Introduction}

Mammalian external genitalia, which show morphological differences among species, are reproductive organs facilitating efficient copulation and internal fertilisation (Yamada et al. 2003, Murashima et al. 2015). Furthermore, external genital malformations, known as human birth defects (i.e. hypospadias and ambiguous external genitalia), are caused by a combination of genetic and environmental factors (Yamada et al. 2006, van der Zanden et al. 2012, Sinclair et al. 2016a). The genital tubercle (GT) is an embryonic primordium of the external genitalia; it differentiates into the penis in males and the clitoris in females (Yamada et al. 2003, 2006, Cohn 2011). GT morphogenesis is divided into two processes: (a) initial outgrowth and (b) sexual dimorphism characterised by the differentiation of mesenchyme and urethra (Suzuki et al. 2002, Yamada et al. 2003). Male GT is masculinised by androgens secreted from the testes (Miyagawa et al. 2009b, Murashima et al. 2015). GT consists of endodermal and ectodermal epithelia and mesenchyme, and epithelial-mesenchymal interactions play an essential role in its development (Haraguchi et al. 2000, Yamada et al. 2003, Ipulan et al. 2016). The entire urethra, without ectoderm contribution, originates from the endoderm (Seifert et al. 2008, Ipulan et al. 2016). In mice, the tube formation of the most urethra occurs through the canalisation process of the urethral plate from the proximal to distal direction, whereas the distal aspect of the urethra and especially the urethral meatus form through fusion of the preputialurethral folds (Seifert et al. 2008, Mahawong et al. 2014, Georgas et al. 2015, Sinclair et al. 2016a,b). Several signalling pathways, including the fibroblast growth factor (FGF) (Haraguchi et al. 2000, Miyagawa et al. 2009a, Lin et al. 2013, Gredler et al. 2015, Harada et al. 2015), sonic hedgehog (SHH) (Haraguchi et al. 2001, Perriton et al. 2002, Lin et al. 2009, Miyagawa et al. 2009a, Seifert et al. 2009a) and bone morphogenetic protein (BMP) (Suzuki et al. 2003, 2008, Wu et al. 2009) pathways, control mouse GT formation. Phenotypic analyses of genetically modified or endocrine-modulated mice in vivo and their GTs 
cultured in vitro partially uncovered the molecular mechanism involved in the embryonic development of external genitalia (Ipulan et al. 2016). However, comparative analyses between mammalian species using various non-rodent models are required to fully understand the mechanisms underlying the morphological diversity observed in the mammalian external genitalia.

The Asian house musk shrew Suncus murinus (hereafter, suncus) belongs to the order Eulipotyphla (the former order Insectivora or Soricomorpha), which is classified into the superorder Laurasiatheria. In contrast, the orders Primates and Rodentia are classified into the superorder Euarchontoglires (Bininda-Emonds et al. 2007). Thus, suncus is a phylogenetically distinct species from mice and humans. Previous studies have reported morphological analysis of the external genitalia of the adult male suncus (Bedford et al. 1997, Kamikawa-Miyado et al. 2005), and Kitoh and coworkers (1985) have reported that the urethral and rectal orifices of the suncus are encompassed by a cavity called the ostium urogenitoanalis. We have elucidated one of the primary characteristic features of the adult suncus external genitalia: the presence of musculus ischiocavernosus dorsalis (KamikawaMiyado et al. 2005). Notably, several morphological features of the external genitalia of the adult male suncus are distinct from those of mouse and human (Table 1) (Glucksmann et al. 1976, Murakami 1987, Yamada et al. 2003, Kamikawa-Miyado et al. 2005, Rodriguez et al. 2012, Weiss et al. 2012, Sinclair et al. 2016c). In both humans and the adult male suncus, the mid-shaft of male external genitalia possesses a penile urethra, a corpus spongiosum urethrae (also known as a corpus cavernosum urethrae) and a corpus cavernosum penis covered with an extremely thick tunica albuginea (Yamada et al. 2003, KamikawaMiyado et al. 2005). The middle part of the adult male mouse external genitalia possesses a penile urethra, a corpus spongiosum urethrae, a circumferential corpus cavernosum glandis and a penile bone (Table 1) (Glucksmann et al. 1976, Murakami 1987, Yamada et al. 2003, Rodriguez et al. 2012, Weiss et al. 2012, Sinclair et al. 2016c). However, the processes involved in the development of the embryonic GT in the suncus remain unclear.

In the present study, we investigated the changes in morphological features and gene expression patterns during the development of the suncus GT.

\section{Materials and methods}

\section{Animals}

All animal experiments were approved by the Animal Care Committee of the National Research Institute for Child Health and Development (project number: A2007-001) and Kumamoto University. All experiments were performed in accordance with the institutional guidelines of the care and use of laboratory animals. Adult female and male suncus of an outbred KAT strain were maintained at $25^{\circ} \mathrm{C}$ under a 12-h light and 12-h darkness cycle, with food (CIEA-305; Clea, Japan) and water ad libitum.

\section{Embryo preparation}

For mating, the female was housed with the male for $2 \mathrm{~h}$. The day after mating was denoted as embryonic day 0 (E0). The gestation period was approximately 30 days, and pregnant females were killed to collect embryos staged at E14-E26. The embryonic stage was estimated according to the morphological features of embryos, as described previously (Inouye et al. 1985). After E20, the sex of the embryo was determined based on the gonadal shape and size. Tissue samples were fixed with $4 \%$ paraformaldehyde (PFA), dehydrated with methanol and stored at $-20^{\circ} \mathrm{C}$ until used in further experiments. Before being frozen in OCT, the tissues were fixed with $4 \%$ PFA and cryoprotected in $20 \%$ sucrose. The main three body axes and the corresponding GT axes are illustrated in Fig. 1.

Table 1 Structural comparison of adult male external genitalia and testis.

\begin{tabular}{llc}
\hline & Suncus & Mouse \\
\hline Orifices of urethra and rectum & Share of a large common cavity (ostium & Separation \\
urogenitoanalis) & Present & Separation \\
Urethra completely within penis & Present & Present \\
Defined erectile bodies & Present & Present \\
Distal-dorsal loose connective tissue & Absent & Absent \\
Distal fibrocartilage & Absent & Present \\
Proximal hyaline cartilage & Absent & Present \\
Penile bone & Present & Present \\
Skeletal muscle within the penile shaft & Absent \\
Pair of smooth muscles within the penile shaft & Present & Absent \\
Extremely thick tunica albuginea & Present & Absent \\
Circumferential penile epithelium & Present & Absent \\
Keratinised epithelial spines & Present & Absent \\
No tethering, freely mobile & Present & Absent \\
Prepuce & Present & Absent \\
Preputial gland & Verification required & Present \\
Testes position & Abdominal cavity & Present \\
\hline
\end{tabular}



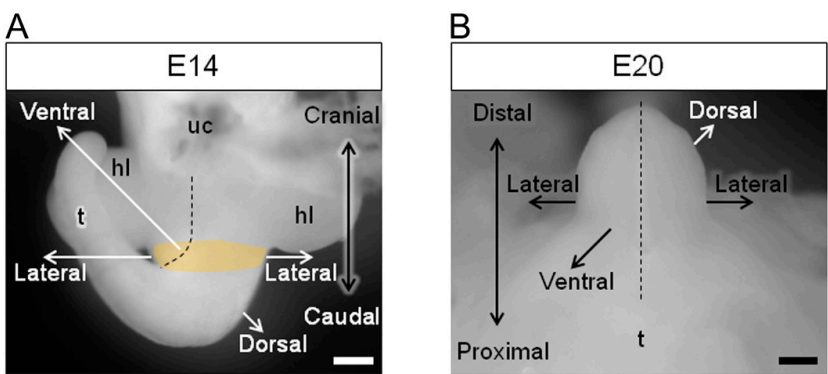

Figure 1 Development of the suncus genital tubercle (GT). (A) The cloacal region before appearance of the GT. (B) Ventral view of the developing GT. Dashed lines show the abdominal median line and the midline of the GT respectively. The orange-coloured region shows the cloacal region. hl, hind limb; t, tail; uc, umbilical cord. Scale bars, $200 \mu \mathrm{m}$.

\section{Scanning electron microscope (SEM) analysis}

Embryos stored at $-20^{\circ} \mathrm{C}$ were critical-point dried after tails were removed, and methanol in the samples was replaced with isoamyl acetate. The treated samples were mounted on an aluminium stub using carbon conductive tape and a silver paste and were coated with gold particles. The sample surface was observed using an SEM (S-800; Hitachi) and photographed using a film camera. At least two embryos were examined for each stage and sex.

\section{Analysis of gene expression patterns}

Whole-mount in situ hybridisation was performed as described previously (Ogi et al. 2002, Miyado et al. 2007). Stained samples were embedded in albumin and sectioned into 30- $\mu$ m-thick slices using a vibratome. At least three embryos were examined for each gene, stage and sex. In situ hybridisation was performed using sections (14- $\mu \mathrm{m})$ of frozen tissue from two male GTs staged at E22, according to standard procedures.

\section{Histological analysis}

Embryos stored at $-20^{\circ} \mathrm{C}$ were immersed in ethanol and xylene, embedded in paraffin and sectioned. Serial 6 - $\mu$ m-thick sections were mounted on slides and stained with hematoxylin-eosin. At least three embryos were examined for each stage and sex.

\section{Results}

\section{Features for initial outgrowth of the suncus external genitalia}

To examine the morphological features of the suncus external genitalia, we performed SEM analysis of the external genitalia from E14 (before the appearance of the GT) to E18 (before sexual differences appeared morphologically; Fig. 2). In E14 embryos, outgrowth of the GT was not detected in the abdominal median line of the embryo (white arrowheads in Fig. 2A). At
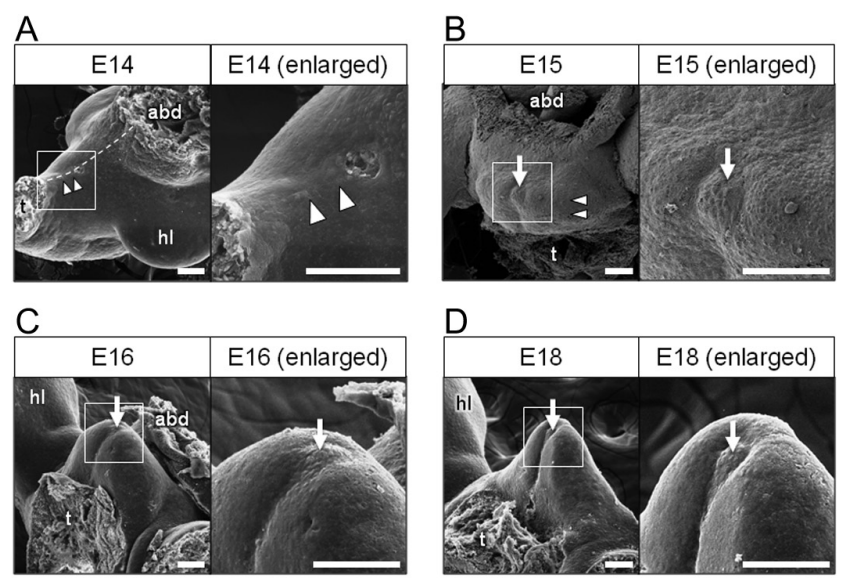

Figure 2 Electron microscopic observation of the genital tubercle (GT) during initial outgrowth. The cloacal region at E14 (A) and outgrowing GT at E15 (B), E16 (C) and E18 (D). Photographs in A, C and $D$ were arranged with the tail bud oriented toward the bottom left of the photo. Photographs in B were arranged with the tail bud oriented toward the bottom of the photo. In A and B, white arrowheads indicate the cloacal region and genital swellings respectively. In B, C and D, white arrows indicate a small distal protrusion at the tip of the GT. The white-dashed line shows the abdominal median line. abd, abdomen; hl, hind limb; t, tail. Scale bars, $100 \mu \mathrm{m}$.

E15, slight GT outgrowth was observed between the abdomen and tail (white arrowheads in Fig. 2B), and the cloaca was divided into a urogenital sinus and rectum. At E16 and E18, the GT was prominently elongated, and a small distal protrusion was observed at its tip (white arrows in Fig. 2C and D). No sex-specific morphological differences were observed in any of the stages examined here.

\section{Emergence of sexual dimorphism of the suncus external genitalia}

We also performed SEM analysis of the GT at E20 and later stages (Fig. 3). At E20, no sex-specific differences were detected at the distal region of the GT, whereas the signs of sexual dimorphism were visualised at the proximal region (white arrows in Fig. 3A1 and A2). The anogenital distance, which is one of the signs of sexual dimorphism, was longer in males than in females. At E22, the proximal region of the GT showed greater sex-specific differences than at E20, and the anogenital distance was more longer in males than that in females (white arrows in Fig. 3A3 and A4). At E24, the glans of the GT was round in males and egg-shaped in females (blue-coloured regions in Fig. $3 \mathrm{~A} 5$ and A6), and the GT was beginning to be engulfed in the preputial skin in both sexes (Fig. 3A5 and A6). At E26, the GT was covered with the skin in both sexes (Fig. 3B1 and B2). The urethral and rectal orifices in adult males and the urethral, rectal and vaginal orifices in adult females share a cavity termed the ostium urogenitoanalis 
A

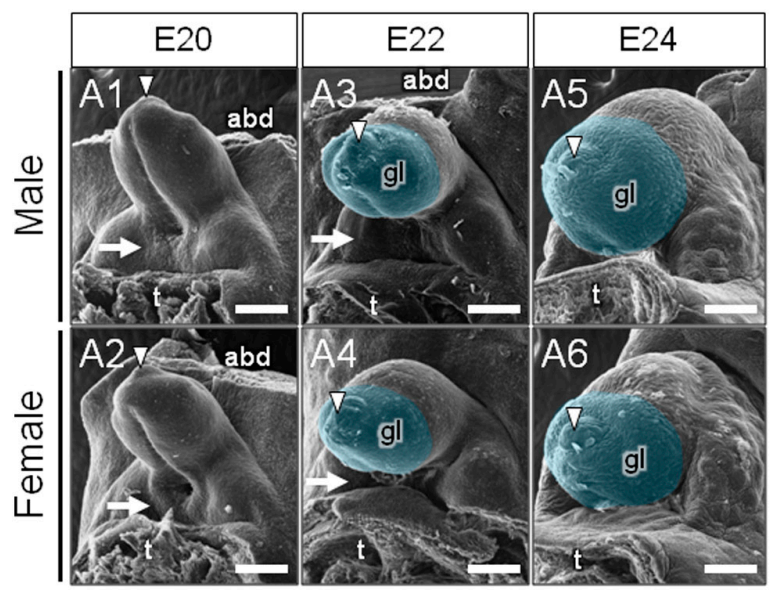

B

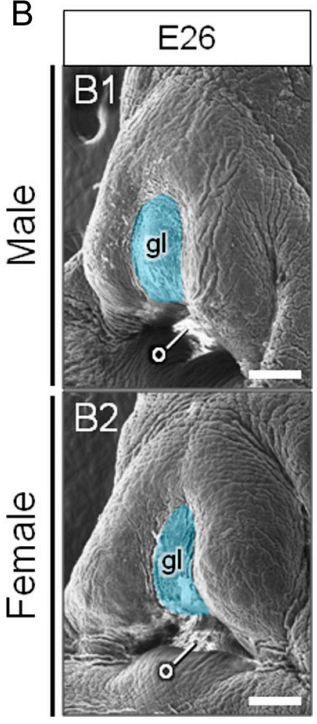

Figure 3 Electron microscopic observation of the genital tubercle (GT) during sexually dimorphic differentiation. Vent-lateral view of the GT samples at E20 (A1 and A2), E22 (A3 and A4), E24 (A5 and A6) and E26 (B1 and B2). All photographs were arranged with the tail bud oriented toward the bottom left of the photo. All GT samples were observed from the left side. Upper and lower panels display male and female GTs at the corresponding stages respectively. White arrowheads indicate the prospective primordium of the distal-dorsal loose connective tissue, which will compose a part of the adult glans in only males. White arrows indicate anogenital distance. Bluecoloured regions show the glans of the GT. abd, abdomen; gl, glans; o, ostium urogenitoanalis; t, tail. Scale bars, $200 \mu \mathrm{m}$.
(Kitoh et al. 1985, Kamikawa-Miyado et al. 2005). At E26, the ostium urogenitoanalis was incompletely formed (Fig. 3B1 and B2).

\section{Gene expression during the suncus GT development}

During the developmental stages of mouse external genitalia, Fgf8 is expressed in the cloacal epithelium and the distal urethral epithelium of the GT and serves as an indicator of budding and outgrowth of the GT (Haraguchi et al. 2000, Ipulan et al. 2014). Therefore, we first examined the expression of $F g f 8$ in the suncus GT (Fig. 4A). In the absence of outgrowth of the GT, Fgf8 was expressed in the abdominal median line, particularly in the cloacal epithelium at E14 (white arrows in Fig. 4A1, A2 and A3 and Supplementary Fig. $1 \mathrm{~B}$ and $\mathrm{C}$, see section on supplementary data given at the end of this article). Furthermore, during GT outgrowth at E15 and E18, Fgf8 was expressed in the distal GT region (white arrows in Fig. 4A4, A5, A6 and A7). At E20, Fgf8 was expressed at the GT tip, but its signal was weak (white arrows in Fig. 4A8 and A9). These expression patterns are similar to those observed during GT outgrowth in mice. Previous studies have shown that Shh is expressed in the endodermal cloacal epithelium and the urethral epithelium and is involved in the dual processes of GT outgrowth and urethral tube formation in mice (Haraguchi et al. 2001,
A
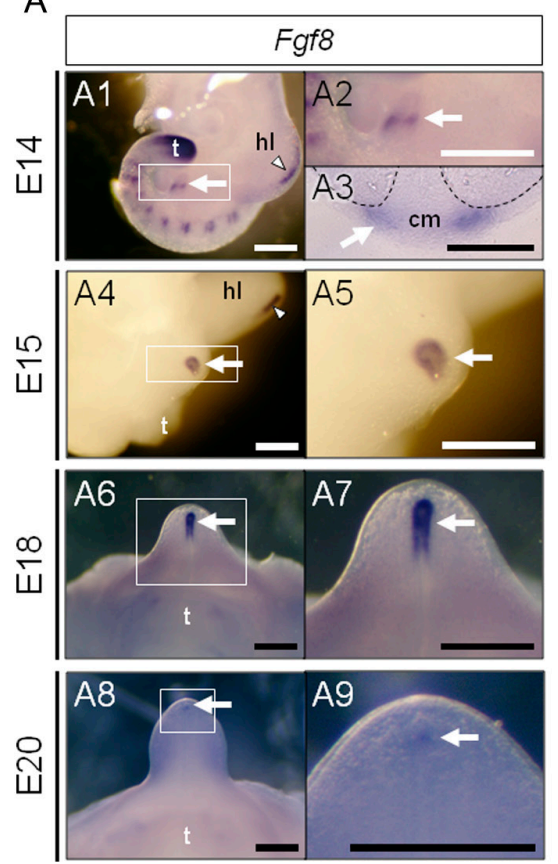

B
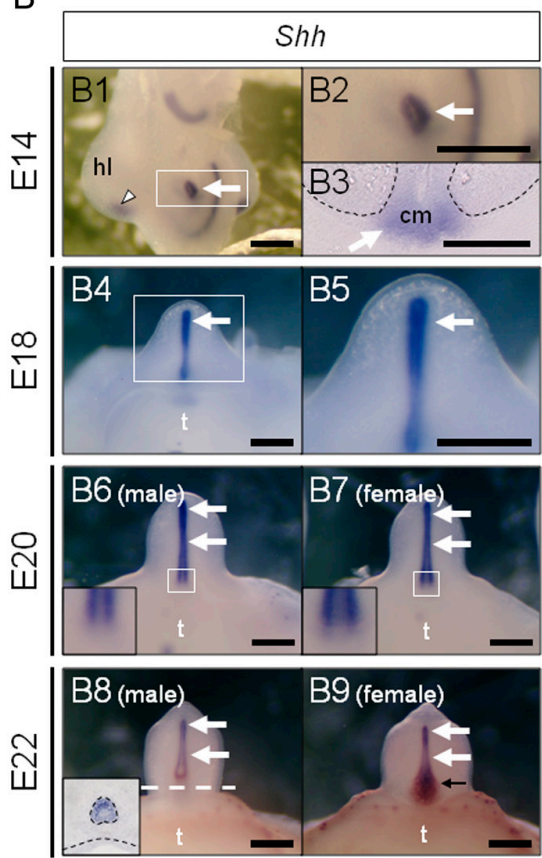

Figure 4 Expression of Fgf8 and Shh in the developing genital tubercle (GT). (A) Whole-mount in situ hybridisation for Fgf8 at E14 (A1, A2 and A3), E15 (A4 and A5), E18 (A6 and A7), and E20 (A8 and A9). (B) Whole-mount in situ hybridisation for Shh at E14 (B1, B2 and B3), E18 (B4 and B5), E20 (B6 and $B 7)$, and E22 (B8 and B9). Right upper panels in $A$ and $B$ are the enlarged views of the left image. A3 and B3 show images of sections generated after in situ hybridisation (Supplementary Fig. 1). Insets of B6 and B7 show enlarged views of the white square areas. Inset of B8 shows an image obtained by in situ hybridisation using tissue sections. White arrowheads indicate Fgf8 or Shh mRNA-expressing regions in the hind limb. White arrows show Fgf8 or Shh mRNAexpressing regions in the cloaca (A1, A2 and $A 3$, and $B 1, B 2$ and $B 3)$ and the developing GT (A4, A5, A6, A7, A8 and A9, and B4, B5, B6, B7, B8 and B9). Dashed lines indicate the epithelial-mesenchymal boundary. The white-dashed line shows the region subjected to in situ hybridisation. cm, cloaca membrane; hl, hind limb; t, tail. Scale bars, $200 \mu \mathrm{m}$. 


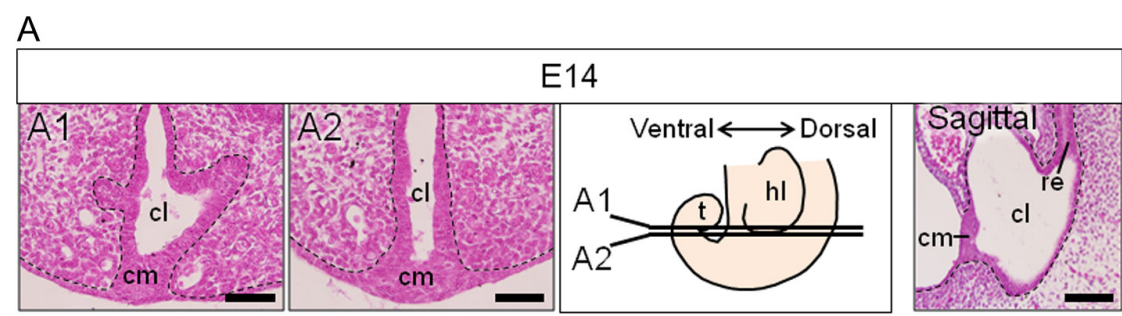

B

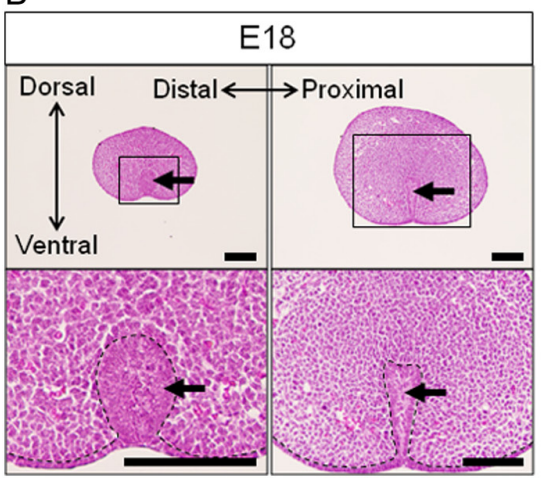

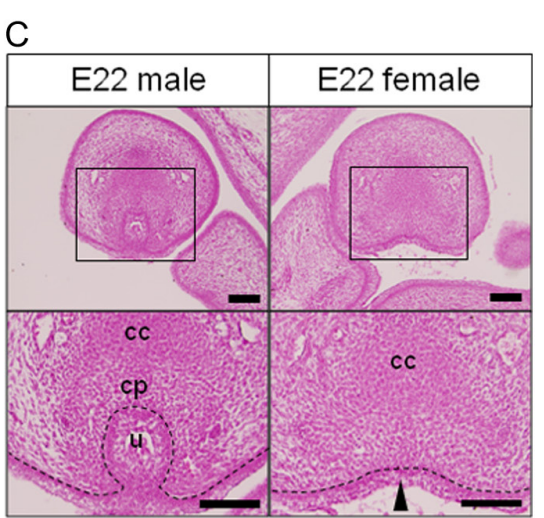

Figure 5 Histological analysis of the developing genital tubercle (GT). (A) Transverse (A1 and A2) and sagittal sections of the cloacal region at E14. (B) Transverse sections of the GT at E18. The left and right panels correspond to the regions of the distal and proximal GT respectively. (C) Transverse sections of the GT at E22. Black-dashed lines indicate the epithelial-mesenchymal boundary. In B, black arrows indicate the urethral plate epithelium. In B and C, the regions enclosed by boxes show the enlarged views of the lower panels. cc, anlage of corpus cavernosum penis or clitoris; $\mathrm{cl}$, cloaca; $\mathrm{cm}$, cloaca membrane; $\mathrm{cp}$, anlage of corpus spongiosum urethrae; hl, hind limb; re, prospective rectum; t, tail; $u$, urethra. Scale bars, $100 \mu \mathrm{m}$.
Miyagawa et al. 2009a). Therefore, we next examined the region expressing Shh in the suncus GT (Fig. 4B). Shh was expressed in the abdominal median line, exclusively in the cloacal epithelium, before GT outgrowth at E14 (white arrows in Fig. 4B1, B2 and B3 and Supplementary Fig. $1 \mathrm{~B}^{\prime}$ and $\mathrm{C}^{\prime}$ ). At E18 and E20, Shh was widely expressed in the ventral midline of the GT from the proximal to distal regions (white arrows in Fig. 4B4, B5, B6 and B7). At E22, Shh was also expressed in the ventral midline of the GT in both sexes (white arrows in Fig. 4B8 and B9), although sex-specific expression pattern was observed in the proximal region. Shh expression was not visible in the proximal side of the male GT (white-dashed line in Fig. 4B8). In contrast, Shh was expressed in the proximal aspect of the female GT (black arrow in Fig. 4B9). Expression of Shh was detected in the urethral tube epithelium on the proximal side of the male GT staged at E22 by detailed additional in situ hybridisation analyses using frozen tissue sections (inset in Fig. 4B8). These expression patterns observed are quite similar to those reported during the outgrowth of the mouse GT and in urethral tube formation in the mouse GT.

\section{Histological features of the suncus GT}

We also conducted histological examination of the developing suncus GT (Fig. 5). At E14, the cloacal membrane was formed by the contact region between ectodermal and endodermal epithelia (Fig. 5A). The ventral cloacal epithelium was thicker than the dorsal cloacal epithelium on the dorsal aspect of the GT. At the distal and proximal regions of the GT, the mesenchymal layers were undifferentiated and primordial, even at E18 (Fig. 5B). The distal urethral plate appeared as more condensed epithelium than the urethral plate at the proximal region of the GT (arrows in Fig. 5B). At $\mathrm{E} 22$, the urethra was already formed as a ductal structure at the proximal region of the male GT (' $u$ ' in Fig. $5 \mathrm{C}$ ), whereas no urethral structure was observed at the proximal region of the female GT (arrowhead in Fig. 5C). At E22, mesenchymal condensations were observed as the prospective corpus cavernosum of the penis and the clitoris (' $\mathrm{CC}^{\prime}$ in Fig. $5 \mathrm{C}$ ), and a mesenchymal condensation surrounding the forming penile urethra was also noted (' $\mathrm{cp}^{\prime}$ ' in Fig. 5C).

\section{Discussion}

In this study, we demonstrated the expression of the essential growth factors Fgf 8 and Shh and characterised the morphological features of the embryonic GT, a common anlage of the external genitalia, of the suncus. We determined the time course of GT development and the emergence of sexual dimorphism, including urethral development. As reported previously in mouse, the GT develops from the cloacal region starting at E10.5 (Haraguchi et al. 2000, Yamada et al. 2006, Georgas et al. 2015), indicating that GT outgrowth is a consequence of mesenchymal swelling around the cloaca. During mouse GT development, Fgf8 is expressed from E10.5, shortly before GT outgrowth, and up to E14.0, when GT outgrowth is prominent; this Fgf8-expressing region is termed the distal urethral epithelium (Haraguchi et al. 2000, Perriton et al. 2002). Removal of this region reduces the expression of mesenchymal genes such as Fgf10, suppressing GT 
outgrowth (Haraguchi et al. 2000). Therefore, interaction between the distal urethral epithelium and its peripheral mesenchymal region is thought to play a major role in both swelling and outgrowth (Haraguchi et al. 2000). As shown in Fig. 2A, the formation of the cloacal membrane was unclear in the abdominal median line in the suncus, whereas the cloacal membrane is formed clearly as a groove in mice (Suzuki et al. 2002). The difference in cloaca morphology emerged before the GT outgrowth, possibly due to the morphological diversity of the external genitalia. Despite the morphological differences between the mouse and suncus, we assumed that the cloacal epithelium may control initial GT outgrowth in the suncus because Fgf8 was expressed in this region. We also observed the small distal protrusion at the tip of the suncus GT, which may correspond to the distal urethral epithelium reported in mouse GT (Haraguchi et al. 2000). The region emerged at E15 and was clearly distinguishable prior to at E20, when Fgf8 was expressed marginally. Fgf8 conditional knockout and Fgf $4 / 8$ compound-mutant mice have been shown to exhibit normal GT outgrowth, although FGF8 induces extracellular signal-regulated kinase 1/2 phosphorylation and cell proliferation in the mouse GT in vitro (Miyagawa et al. 2009a, Seifert et al. 2009b). In mouse GT development, the presence of regulatory mechanisms overlapped among multiple FGFs, which are functionally redundant from phenotypic analyses of their knockout mice, has been discussed (Miyagawa et al. 2009a). These findings indicate that FGF signalling serves as an important regulator of GT outgrowth not only in the mouse but also in the suncus. Because redundant and outgrowth-regulating functions of FGFs have been well studied in the mouse GT (Miyagawa et al. 2009a), the current finding indicates a potentially intriguing possibility of similar/divergent functions of FGF signals in the suncus, which should necessarily be examined by further studies.

As shown in Fig. 4B, Shh was expressed in the cloacal epithelium and the developing urethral epithelium of the suncus GT, and its expression pattern is quite similar to that in the mouse GT. In the ventral midline of the mouse GT, endodermal and ectodermal epithelia and the mesenchyme contact each other, and the urethral tube except for the distal aspect of the urethra is formed by canalisation of the urethral plate (Seifert et al. 2008, Miyagawa et al. 2009b, Herrera \& Cohn 2014, Mahawong et al. 2014, Sinclair et al. $2016 a, b)$. In fact, a tubular urethra was recognised within the proximal urethral plate epithelium of the male suncus GT staged at E22. Although the tube formation of the distal aspect of the urethra needs to be validated by further studies, it is likely that the most urethra of the suncus may be formed in a direction from the proximal urethral plate to the distal plate, similar to that in the mouse (Seifert et al. 2008, Mahawong et al. 2014, Georgas et al. 2015, Sinclair et al. 2016a,b).
In mice, several growth factors have been reported to control GT outgrowth and urethral tube formation (Yamada et al. 2006, Ipulan et al. 2016). For example, Shh is expressed in the cloacal and urethral epithelium of the GT and plays critical roles in both GT outgrowth initiation and mesenchymal differentiation of the GT (Haraguchi et al. 2001, 2007, Perriton et al. 2002, Miyagawa et al. 2009a). SHH signalling is also involved in the patterning and development of the male urethra in humans (Shehata et al. 2011). Our results indicate that $\mathrm{SHH}$ plays similar roles in the mouse and the suncus during GT development.

The suncus has been increasingly used as a model species to analyse organogenesis including genitalia, craniofacial and tooth development during the prenatal period (Niida et al. 1994, Ogi et al. 2002, Miyado et al. 2007, Yamanaka et al. 2007). Epithelial-mesenchymal interactions widely promote embryogenesis and also GT and tooth development in mice. Owing to the morphological diversity, different molecular mechanisms exist in the GT and tooth germ (Thesleff 2003). However, SHH signalling is required for the early development and patterning of both GT and tooth germ, and the expression patterns of many genes in both organs are quite similar. As described previously (Miyado et al. 2007, Yamanaka et al. 2007), the suncus tooth germ expresses Shh, indicating that $\mathrm{SHH}$ function in tooth development is widely conserved among mammals. As reported previously for Shh-expressing organs in mice, $\mathrm{SHH}$ signalling presumably plays a critical role in the development of suncus organs, at least teeth and the GT.

As gene expression in mesenchymal cells was not analysed in this study, the genes associated with mesenchymal differentiation in the suncus GT remain unknown. Mesenchymal differentiation is an indispensable process for sexual dimorphism in the mouse GT (Miyagawa et al. 2009b). The developmental process of external genitalia has been elucidated from studies in rodents, mainly mice (Haraguchi et al. 2000, Suzuki et al. 2003, Yamada et al. 2006, Georgas et al. 2015, Ipulan et al. 2016). Bone is present in the penis (os penis) and clitoris (os clitoris) of mice, whereas only male rats possess this bone (Glucksmann et al. 1976, Murakami 1987, Yamada et al. 2003, Rodriguez et al. 2012, Weiss et al. 2012, Sinclair et al. 2016c). The os penis plays a role in maintaining erection during copulation, because hypoplasia of the os penis are observed in infertile Hoxa13- and Hoxd13-deficient mice (Dollé et al. 1993, Post \& Innis 1999) and males with a wider os penis achieve higher reproductive success than males with a narrower one in mice (Stockley et al. 2013, Schultz et al. 2016). In contrast, there is no corresponding structure in the suncus and humans (Yamada et al. 2003, Kamikawa-Miyado et al. 2005). As the corpus cavernosum penis, which is covered with an extremely thick tunica albuginea, exists 
in the male external genitalia of the suncus and humans (Yamada et al. 2003, Kamikawa-Miyado et al. 2005), we presumed that its function may be comparable to that of the os penis, indicating the unique utility of this species in reproductive/copulation biology. In addition, the external genitalia of the male suncus possesses a muscular structure, which we proposed as the musculus ischiocavernosus dorsalis (Kamikawa-Miyado et al. 2005); this structure is absent in humans and mice. As described, the internal inner structures within of the external genitalia of adult animals have interspecies differences, indicating that the differentiation of the GT is regulated in a species-specific manner. In other words, the genes expressed during the development of external genitalia, especially in GT mesenchymal cells, and the molecular mechanisms driving this process may be species specific. Thus, phylogenetically distinct species, especially non-rodent animals such as the suncus, would be potentially useful models for studying morphological diversity in the mammalian external genitalia. Several genes, including those encoding molecules in the BMP signalling pathway, Wnt/B-catenin, Fgf10-Fgfr2, EphB2-EphrinB2 and Hoxa/Hoxd are involved in the control of mouse GT development (Cohn 2011, Blaschko et al. 2012, Ipulan et al. 2014, Gredler et al. 2015). Furthermore, the time window of androgen actions during the mouse GT masculinisation is from E15.5 to 16.5, and the sexually dimorphic processes are controlled by cross-talk between androgen signalling and growth factors in mice (Miyagawa et al. 2009b, Ipulan et al. 2014), implying that cross-talk between androgen signalling and growth factors presumably plays a role in the morphological diversity. However, some experimental limitations existed in the present study, and further morphological and molecular studies regarding the suncus GT development are being performed by our group and others. Further studies are necessary to clarify what genes and mechanisms such as androgen-triggered mechanism are associated with the development of the suncus GT.

In summary, we demonstrated here that the expression patterns of growth factors Fgf8 and Shh in the suncus GT were similar to those in the mouse GT and that there were species-specific morphological characteristics in the suncus GT, although the two genes examined in this study exhibited parallel expression patterns in both species. Our findings provide insights into mammalian GT development. To fully understand the mechanism of GT morphogenesis, further examination of the expression patterns of other genes that contribute to the development of the suncus GT is needed.

\section{Supplementary data}

This is linked to the online version of the paper at http://dx.doi. org/10.1530/REP-16-0231.

\section{Declaration of interest}

The authors declare that there is no conflict of interest that could be perceived as prejudicing the impartiality of the research reported.

\section{Funding}

This study was supported by a grant from the Ministry of Health, Labour and Welfare and a Grant-in-aid for Scientific Research (C) (grant number 16K08603) from the Ministry of Education, Culture, Sports, Science, and Technology of Japan.

\section{Acknowledgements}

The authors thank Dr Ichiro Sakata, Dr Takafumi Sakai, Dr Hidenao Ogi, Dr Hironori Katoh and Dr Yukiko Ogino for their suggestions and encouragement. They would like to express their gratitude to Editage (www.editage.jp) for English language editing.

\section{References}

Bedford JM, Mori T \& Oda S 1997 Ovulation induction and gamete transport in the female tract of the musk shrew, Suncus murinus. Journal of Reproduction and Fertility 110 115-125. (doi:10.1530/ jrf.0.1100115)

Bininda-Emonds OR, Cardillo M, Jones KE, MacPhee RD, Beck RM, Grenyer R, Price SA, Vos RA, Gittleman JL \& Purvis A 2007 The delayed rise of present-day mammals. Nature 446 507-512. (doi:10.1038/ nature05634)

Blaschko SD, Cunha GR \& Baskin LS 2012 Molecular mechanisms of external genitalia development. Differentiation 84 261-268. (doi:10.1016/j.diff.2012.06.003)

Cohn MJ 2011 Development of the external genitalia: conserved and divergent mechanisms of appendage patterning. Developmental Dynamics 240 1108-1115. (doi:10.1002/dvdy.22631)

Dollé P, Dierich A, LeMeur M, Schimmang T, Schuhbaur B, Chambon P \& Duboule D 1993 Disruption of the Hoxd-13 gene induces localized heterochrony leading to mice with neotenic limbs. Cell 75 431-441. (doi:10.1016/0092-8674(93)90378-4)

Georgas KM, Armstrong J, Keast JR, Larkins CE, McHugh KM, SouthardSmith EM, Cohn MJ, Batourina E, Dan H, Schneider K et al. 2015 An illustrated anatomical ontology of the developing mouse lower urogenital tract. Development 142 1893-1908. (doi:10.1242/ dev.117903)

Glucksmann A, Ooka-Souda S, Miura-Yasugi E \& Mizuno T 1976 The effect of neonatal treatment of male mice with antiandrogens and of females with androgens on the development of the os penis and os clitoridis. Journal of Anatomy 121 363-370.

Gredler ML, Seifert AW \& Cohn MJ 2015 Tissue-specific roles of Fgfr2 in development of the external genitalia. Development 142 2203-2212. (doi:10.1242/dev.119891)

Harada M, Omori A, Nakahara C, Nakagata N, Akita K \& Yamada G 2015 Tissue-specific roles of FGF signaling in external genitalia development. Developmental Dynamics 244 759-773. (doi:10.1002/dvdy.24277)

Haraguchi R, Suzuki K, Murakami R, Sakai M, Kamikawa M, Kengaku M, Sekine K, Kawano H, Kato S, Ueno N et al. 2000 Molecular analysis of external genitalia formation: the role of fibroblast growth factor (Fgf) genes during genital tubercle formation. Development 127 2471-2479.

Haraguchi R, Mo R, Hui C, Motoyama J, Makino S, Shiroishi T, Gaffield W \& Yamada G 2001 Unique functions of Sonic hedgehog signaling during external genitalia development. Development 128 4241-4250. 
Haraguchi R, Motoyama J, Sasaki H, Satoh Y, Miyagawa S, Nakagata N, Moon A \& Yamada G 2007 Molecular analysis of coordinated bladder and urogenital organ formation by Hedgehog signaling. Development 134 525-533. (doi:10.1242/dev.02736)

Herrera AM \& Cohn MJ 2014 Embryonic origin and compartmental organization of the external genitalia. Scientific Reports 46896. (doi:10.1038/srep06896)

Inouye M, Oda S, Shimamura K \& Kameyama Y 1985 Suncus murinus: Biology of the Laboratory Shrew, pp 140-143. Ed K Kondo. Tokyo (In Japanese): Japan Scientific Societies Press.

Ipulan LA, Suzuki K, Matsushita S, Suzuki H, Okazawa M, Jacinto S, Hirai S \& Yamada G 2014 Development of the external genitalia and their sexual dimorphic regulation in mice. Sexual Development 8 297-310. (doi:10.1159/000357932)

Ipulan LA, Raga D, Suzuki K, Murashima A, Matsumaru D, Cunha G \& Yamada G 2016 Investigation of sexual dimorphisms through mouse models and hormone/hormone-disruptor treatments. Differentiation 91 78-89. (doi:10.1016/j.diff.2015.11.001)

Kamikawa-Miyado M, Ogi H, Ogino Y, Katoh H, Suzuki K, Uemura M, Kitoh J, Oda S \& Yamada G 2005 The morphological and histological characters of the male external genitalia of the house musk shrew, Suncus murinus. Zoological Science 22 463-468. (doi:10.2108/ zsj.22.463)

Kitoh J, Ohta K, Yamashita K, Sugiura Y, Hirunagi K, Oda S \& Yohoyama A 1985 Suncus murinus: Biology of the Laboratory Shrew, pp 239-258. Ed K Kondo. Tokyo (In Japanese): Japan Scientific Societies Press.

Lin C, Yin Y, Veith GM, Fisher AV, Long F \& Ma L 2009 Temporal and spatial dissection of Shh signaling in genital tubercle development. Development 136 3959-3967. (doi:10.1242/dev.039768)

Lin C, Yin Y, Bell SM, Veith GM, Chen H, Huh SH, Ornitz DM \& Ma L 2013 Delineating a conserved genetic cassette promoting outgrowth of body appendages. PLoS Genetics 9 e1003231. (doi:10.1371/journal. pgen.1003231)

Mahawong P, Sinclair A, Li Y, Schlomer B, Rodriguez E Jr, Ferretti MM, Liu B, Baskin LS \& Cunha GR 2014 Comparative effects of neonatal diethylstilbestrol on external genitalia development in adult males of two mouse strains with differential estrogen sensitivity. Differentiation 88 70-83. (doi:10.1016/j.diff.2014.09.004)

Miyado M, Ogi H, Yamada G, Kitoh J, Jogahara T, Oda S, Sato I, Miyado K \& Sunohara M 2007 Sonic hedgehog expression during early tooth development in Suncus murinus. Biochemical and Biophysical Research Communications 363 269-275. (doi:10.1016/j. bbrc.2007.08.158)

Miyagawa S, Moon A, Haraguchi R, Inoue C, Harada M, Nakahara C, Suzuki K, Matsumaru D, Kaneko T, Matsuo I et al. 2009a Dosage-dependent hedgehog signals integrated with Wnt/betacatenin signaling regulate external genitalia formation as an appendicular program. Development 136 3969-3978. (doi:10.1242/ dev.039438)

Miyagawa S, Satoh Y, Haraguchi R, Suzuki K, Iguchi T, Taketo MM, Nakagata N, Matsumoto T, Takeyama K, Kato S et al. 2009b Genetic interactions of the androgen and Wnt/beta-catenin pathways for the masculinization of external genitalia. Molecular Endocrinology 23 871-880. (doi:10.1210/me.2008-0478)

Murakami R 1987 A histological study of the development of the penis of wild-type and androgen-insensitive mice. Journal of Anatomy 153 223-231.

Murashima A, Kishigami S, Thomson A \& Yamada G 2015 Androgens and mammalian male reproductive tract development. Biochimica et Biophysica Acta 1849 163-170. (doi:10.1016/j.bbagrm. 2014.05.020)

Niida S, Okada N, Wakisaka H, Miyata K \& Maeda N 1994 Occipital roof development in the Japanese musk shrew, Suncus murinus. Journal of Anatomy 185 433-437.

Ogi H, Tabata MJ, Yamanaka A, Yasui K \& Uemura M 2002 Comparison of expression patterns of fibroblast growth factor 8 , bone morphogenetic protein 4 and sonic hedgehog in jaw development of the house shrew, Suncus murinus. Cellular and Molecular Biology 48 OL289-OL296.

Perriton CL, Powles N, Chiang C, Maconochie MK \& Cohn MJ 2002 Sonic hedgehog signaling from the urethral epithelium controls external genital development. Developmental Biology 247 26-46. (doi:10.1006/ dbio.2002.0668)

Post LC \& Innis JW 1999 Infertility in adult hypodactyly mice is associated with hypoplasia of distal reproductive structures. Biology of Reproduction 61 1402-1408. (doi:10.1095/biolreprod61.6.1402)

Rodriguez E Jr, Weiss DA, Ferretti M, Wang H, Menshenia J, Risbridger G, Handelsman D, Cunha G \& Baskin L 2012 Specific morphogenetic events in mouse external genitalia sex differentiation are responsive/ dependent upon androgens and/or estrogens. Differentiation $\mathbf{8 4}$ 269-279. (doi:10.1016/j.diff.2012.07.003)

Schultz NG, Ingels J, Hillhouse A, Wardwell K, Chang PL, Cheverud JM, Lutz C, Lu L, Williams RW \& Dean MD 2016 The genetic basis of baculum size and shape variation in mice. G3 6 1141-1151. (doi:10.1534/g3.116.027888)

Seifert AW, Harfe BD \& Cohn MJ 2008 Cell lineage analysis demonstrates an endodermal origin of the distal urethra and perineum. Developmental Biology 318 143-152. (doi:10.1016/j.ydbio.2008.03.017)

Seifert AW, Bouldin CM, Choi KS, Harfe BD \& Cohn MJ 2009a Multiphasic and tissue-specific roles of sonic hedgehog in cloacal septation and external genitalia development. Development 136 3949-3957. (doi:10.1242/dev.042291)

Seifert AW, Yamaguchi T \& Cohn MJ 2009b Functional and phylogenetic analysis shows that Fgf8 is a marker of genital induction in mammals but is not required for external genital development. Development 136 2643-2651. (doi:10.1242/dev.036830)

Shehata BM, Elmore JM, Bootwala Y, Steelman CK, Bare JB, Shoffeitt CJ, Wang R, Zhau HE, He D, Zhu G et al. 2011 Immunohistochemical characterization of sonic hedgehog and its downstream signaling molecules during human penile development. Fetal and Pediatric Pathology 30 244-251. (doi:10.3109/15513815. 2011.555809)

Sinclair AW, Cao M, Baskin L \& Cunha GR 2016a Diethylstilbestrolinduced mouse hypospadias: 'window of susceptibility'. Differentiation 91 1-18. (doi:10.1016/j.diff.2016.01.004)

Sinclair AW, Cao M, Shen J, Cooke P, Risbridger G, Baskin L \& Cunha GR 2016b Mouse hypospadias: a critical examination and definition. Differentiation In press. (doi:10.1016/j. diff.2016.03.004)

Sinclair AW, Glickman SE, Baskin L \& Cunha GR 2016c Anatomy of mole external genitalia: setting the record straight. Anatomical Record 299 385-399. (doi:10.1002/ar.23309)

Stockley P, Ramm SA, Sherborne AL, Thom MD, Paterson S \& Hurst JL 2013 Baculum morphology predicts reproductive success of male house mice under sexual selection. BMC Biology 11 66. (doi:10.1186/17417007-11-66)

Suzuki K, Ogino Y, Murakami R, Satoh Y, Bachiller D \& Yamada G 2002 Embryonic development of mouse external genitalia: insights into a unique mode of organogenesis. Evolution and Development 4 133-141. (doi:10.1046/j.1525-142X.2002.01061.x)

Suzuki K, Bachiller D, Chen YP, Kamikawa M, Ogi H, Haraguchi R, Ogino Y, Minami Y, Mishina Y, Ahn K et al. 2003 Regulation of outgrowth and apoptosis for the terminal appendage: external genitalia development by concerted actions of BMP signaling. Development 130 6209-6220. (doi:10.1242/dev.00846)

Suzuki K, Haraguchi R, Ogata T, Barbieri O, Alegria O, Vieux-Rochas M, Nakagata N, Ito M, Mills AA, Kurita T et al. 2008 Abnormal urethra formation in mouse models of split-hand/split-foot malformation type 1 and type 4. European Journal of Human Genetics 16 36-44. (doi:10.1038/sj.ejhg.5201925)

Thesleff I 2003 Epithelial-mesenchymal signalling regulating tooth morphogenesis. Journal of Cell Science 116 1647-1648. (doi:10.1242/ jcs.00410)

van der Zanden LF, van Rooij IA, Feitz WF, Franke B, Knoers NV \& Roeleveld N 2012 Aetiology of hypospadias: a systematic review of genes and environment. Human Reproduction Update 18 260-283. (doi:10.1093/humupd/dms002)

Weiss DA, Rodriguez E Jr, Cunha T, Menshenina J, Barcellos D, Chan LY, Risbridger G, Baskin L \& Cunha G 2012 Morphology of the external genitalia of the adult male and female mice as an endpoint of sex differentiation. Molecular and Cellular Endocrinology 354 94-102. (doi:10.1016/j.mce.2011.08.009) 
Wu X, Ferrara C, Shapiro E \& Grishina I 2009 Bmp7 expression and nul phenotype in the urogenital system suggest a role in re-organization of the urethral epithelium. Gene Expression Patterns 9 224-230. (doi:10.1016/j.gep.2008.12.005)

Yamada G, Satoh Y, Baskin LS \& Cunha GR 2003 Cellular and molecular mechanisms of development of the external genitalia. Differentiation 71 445-460. (doi:10.1046/j.1432-0436.2003.7108001.x)

Yamada G, Suzuki K, Haraguchi R, Miyagawa S, Satoh Y, Kamimura M Nakagata N, Kataoka H, Kuroiwa A \& Chen Y 2006 Molecular genetic cascades for external genitalia formation: an emerging organogenesis program. Developmental Dynamics 235 1738-1752. (doi:10.1002/ dvdy.20807)
Yamanaka A, Yasui K, Sonomura T \& Uemura M 2007 Development of heterodont dentition in house shrew (Suncus murinus). European Journal of Oral Sciences 115 433-440. (doi:10.1111/j.16000722.2007.00499.x)

Received 27 April 2016

First decision 17 June 2016

Revised manuscript received 10 November 2016

Accepted 14 November 2016 